\title{
Indirect Adaptive Regulator Design Based on TSK Fuzzy Models
}

\author{
Chang-Woo Park*, Jun-Hyuk Choi*, Ha-Gyeong Sung
}

*Korea Electronics Technology Institute

\begin{abstract}
In this paper, we have proposed a new adaptive fuzzy control algorithm based on Takagi-Sugeno fuzzy model. The regulation problem for the uncertain SISO nonlinear system is solved by the proposed algorithm. Using the advanced stability theory, the stability of the state, the control gain and the parameter approximation error is proved. Unlike the existing feedback linearization based methods, the proposed algorithm can guarantee the global stability in the presence of the singularity in the inverse dynamics of the plant. The performance of the proposed algorithm is demonstrated through the problem of balancing and swing-up of an inverted pendulum on a cart.
\end{abstract}

Key Words : Fuzzy control, adaptive control, Takagi-Sugeno model, nonlinear system

\section{Introduction}

Since Mamdani has applied fuzzy control to a steam engine, many successful applications have been reported [1]-[4] In the early stage of fuzzy control, fuzzy control was a direct method for controlling a system without the need of a mathematical model, in contrast to the classical control which is an indirect method with a mathematical model. However, the stability analysis of the indirect or 'model-free' method is difficult and apt to yield a very conservative result. For this reason, the latest fuzzy control researches have been focused on the indirect fuzzy control method.

In the indirect fuzzy control method, the fuzzy system is used to estimate and approximate the plant dynamics. For mathematical simplicity of analysis, singleton fuzzy system or Takagi-Sugeno fuzzy model is frequently used [5]. Theoretically, the fuzzy system can exactly approximate the dynamics of a class of nonlinear plants with the large or infinite number of rules. However, in the practical situations of limited number of rules and incorrect approximation, uncertainties are inevitably produced. To overcome the effect of uncertainties on stability, the various adaptive fuzzy control algorithms are proposed [6]-[15].

Most of the existing adaptive fuzzy control algorithms are based on the feedback linearization method [9]-[15]. However, the feedback linearization method can not be applied to the plant with the singularity in the inverse dynamics. The adaptive fuzzy control algorithms based on the feedback linearization method need the infinite control input, when the state is at singularity of the inverse dynamics or the parameter approximation error diverges to infinity.

To avoid the need of the infinite control input, the existing feedback linearization based methods require the assumption that the state is away from the singularity and the projection algorithm which prevents the parameter approximation error from diverging to infinity.

Manuscript received Dec. 6, 2005; revised Mar. 7, 2006.
In this paper, We have proposed the indirect adaptive fuzzy state feedback regulator using Takagi-Sugeno_Kang(TSK) fuzzy model. The proposed method is less sensitive to singularity than the adaptive fuzzy control algorithms based on the feedback linearization method. And it can guarantee the boundedness of the state and the parameter approximation error directly in the face of the bounded approximation error and external disturbance and we have also proved that the system state converges to equilibrium.

\section{Problem Formulation}

Consider the regulation problem of the following $n$-th order nonlinear SISO system.

$$
x^{(n)}=f(x)+g(x) u+d(t)
$$

where $f$ and $g$ are unknown (uncertain) but bounded continuous nonlinear functions and $d(t)$ denotes the external disturbance which is unknown but bounded in magnitude. Also, $u$ denotes the control input. Let $x=\left[x, \quad \bar{x}, \cdots, x^{(n-1)}\right]^{T} \in R^{n}$ be the state vector of the system which is assumed to be available.

In this paper, well-known Takagi-Sugeno-Kang fuzzy model is used to identify the unknown nonlinear system (1). Takagi-Sugeno-Kang fuzzy model is available in IF-THEN form (2) or Input-Output form (3).

- IF-THEN form

plant rule $i$ :

IF $x$ is $M_{i 1}$ and $x$ is $M_{i 2}$ and $\cdots$ and $x^{(n-1)}$ is $M_{\text {in }}$

THEN $x^{(n)}=a_{i}{ }^{T} x+b_{i} u, i=1,2, \cdots, r$

where $x=\left[x, \dot{x}, \cdots, x^{(n-1)}\right]^{T} \in R^{n}, a_{i} \in R^{n}$, $b_{i} \in R$

$M_{i j}$ is the fuzzy set and $r$ is the number of rules.

- Input-Output form 


$$
\begin{aligned}
x^{(n)} & =\frac{\sum_{i=1}^{r} w_{i}(x)\left\{a_{i}{ }^{T} x+b_{i} u\right\}}{\sum_{i=1}^{r} w_{i}(x)} \\
& =\sum_{i=1}^{r} h_{i}(x)\left\{\boldsymbol{a}_{i}{ }^{T} \boldsymbol{x}+b_{i} u\right\}
\end{aligned}
$$

where $w_{i}(x)=\prod_{j=1}^{n} M_{i j}\left(x^{(j-1)}\right), \quad h_{i}(x)=\frac{w_{i}(x)}{\sum_{i=1}^{r} w_{i}(x)}$

$M_{i j}\left(x^{(j-1)}\right)$ is the grade of membership of $x^{(j-1)}$ in $M_{i j}$. It is assumed in this paper that

$$
w_{i}(x) \geq 0, i=1,2, \cdots, r, \quad \sum_{i=1}^{r} w_{i}(x)>0
$$

Therefore, the following properties hold.

$$
h_{i}(x) \geq 0, i=1,2, \cdots, r, \quad \sum_{i=1}^{r} h_{i}(x)=1
$$

By defining the parameter vectors $\theta_{a}$ and $\theta_{b}$ as

$$
\theta_{a}=\left[\begin{array}{llll}
a_{1}^{T} & a_{2}^{T} & \cdots & a_{r}^{T}
\end{array}\right]^{T} \in R^{n r} \text { and }
$$$$
\boldsymbol{\theta}_{\boldsymbol{b}}=\left[\begin{array}{llll}
b_{1} & b_{2} & \cdots & b_{r}
\end{array}\right]^{T} \in R^{r} \text {, }
$$

Input-Output form (3) can be expressed by the following equivalent equations.

$$
\begin{aligned}
& x^{(n)}=\hat{f}\left(x \mid \theta_{a}\right)+\hat{g}\left(x \mid \theta_{b}\right) u+d(t) \\
& f\left(x \mid \theta_{a}\right)=\theta_{a}{ }^{T} \xi_{a}=\sum_{i=1}^{r} h_{i}(x) a_{i}{ }^{T} x \\
& \hat{g}\left(x \mid \theta_{b}\right)=\theta_{b}{ }^{T} \xi_{b}=\sum_{i=1}^{r} h_{i}(x) b_{i} u
\end{aligned}
$$

where,

$$
\begin{aligned}
& \xi_{a}=\left[\begin{array}{llllll}
h_{1}(x) & x^{T} h_{2}(x) & x^{T} & \cdots & h_{r}(x) & x^{T}
\end{array}\right]^{T} \in R^{n r} \\
& \xi_{b}=\left[\begin{array}{llll}
h_{1}(x) & h_{2}(x) & \cdots & h_{r}(x)
\end{array}\right]^{r} \in R^{r}
\end{aligned}
$$

\section{Indirect adaptive fuzzy state feedback regulator}

First, we define the optimal parameter vectors as

$$
\begin{aligned}
& \boldsymbol{\theta}_{a}^{*}=\arg \min _{\boldsymbol{\theta}_{a} \in R^{n r}}\left[\sup _{x \in R^{n}}\left|f\left(x \mid \theta_{a}\right)-f(x)\right|\right] \\
& \boldsymbol{\theta}_{b}^{*}=\arg \min _{\theta_{b} \in R^{r}}\left[\sup _{x \in R^{n}}\left|\hat{g}\left(x \mid \theta_{b}\right)-g(x)\right|\right] \\
& \text { where } \theta_{a}^{*}=\left[\begin{array}{lllll}
a_{1}^{* T} & a_{2}^{* T} & \cdots & a_{r}^{* T}
\end{array}\right]^{T} \in R^{n r} \text {, } \\
& \boldsymbol{\theta}_{b}^{*}=\left[\begin{array}{llll}
b_{1}^{*} & b_{2}^{*} & \cdots & b_{r}^{*}
\end{array}\right]^{T} \in R^{r}
\end{aligned}
$$

Thus $f\left(x \mid \theta_{a}^{*}\right)$ and $\hat{g}\left(x \mid \boldsymbol{\theta}_{b}^{*}\right)$ are the optimal approximators of $f(x)$ and $g(x)$, respectively, among all the fuzzy systems in the form of (4-2) and (4-3). However, in spite of the optimal approximation, uncertainties are inevitably produced in the practical application. Considering uncertainties, the uncertain system (1) can be represented by the optimal approximations as in the following equations.

$$
x^{(n)}=f(x)+g(x) u+d(t)
$$

$$
\begin{gathered}
f(x)=f\left(x \mid \theta_{a}^{*}\right)+\Delta a(t)^{T} x \\
=\sum_{i=1}^{r} h_{i}(x) a_{i}^{* T} x+\Delta a(t)^{T} x \\
g(x)=\hat{g}\left(x \mid \theta_{b}^{*}\right)+\Delta b(t) \\
=\sum_{i=1}^{r} h_{i}(x) \quad b_{i}^{*} u+\Delta b(t)
\end{gathered}
$$

where $\Delta a(t) \in R^{n} \quad$ and $\quad \Delta b(t) \in R \quad$ denote the time-varying uncertainties which are assumed to be bounded by some known $\Delta a$ and $\Delta b$ as follows.

$$
\begin{aligned}
& \Delta a=\left[\begin{array}{llll}
\Delta a_{1} & \Delta a_{2} & \cdots & \Delta a_{n}
\end{array}\right]^{T} \in R^{n+} \\
& \left|\Delta a_{j}(t)\right| \leq \Delta a_{j}, \text { for all } j \\
& \Delta b \in R^{+} \\
& |\Delta b(t)| \leq \Delta b
\end{aligned}
$$

Also, the external disturbance is assumed to be bounded by some known $d$.

$$
d \in R^{+}, \quad|d(t)| \leq d
$$

To regulate the uncertain system (6-1) - (6-3), we propose the following indirect adaptive fuzzy state feedback regulator $(7-1)-(7-4)$.

$$
u=k^{T} x
$$

with

$$
\begin{gathered}
k=-\Lambda \hat{T}\left(x, k, \widetilde{a}_{i}, \varpi_{i}, t\right) P x \\
\dot{a}_{i}{ }^{T}=\alpha_{1} h_{i}(x) x^{T} P w x^{T} \\
b_{i}=\alpha_{2} h_{i}(x) x^{T} P w k^{T} x
\end{gathered}
$$

where,

i) $\widetilde{a}_{i}$ and $\widetilde{b}_{i}$ are the parameter approximation errors defined as

$\widetilde{a}_{i}=a_{i}^{*}-a_{i}, \quad \boldsymbol{\delta}_{i}=b_{i}^{*}-b_{i}$ for all $i$, respectively.

ii) $k \in R^{n}$ is the adaptive state feedback gain vector. $\Lambda$ is any $n \times n$ symmetric positive definite adaptation gain matrix. $\alpha_{1}$ and $\alpha_{2}$ are the positive adaptation gain constants.

iii) $\boldsymbol{P}$ is the $n \times n$ symmetric positive definite solution of the Lyapunov equation

$$
\underline{A}^{T} P+P \underline{A}=-Q,
$$

with $Q$ any symmetric positive definite matrix.

In this paper, $\boldsymbol{A}$ is chosen to satisfy that

$$
\begin{aligned}
& \underline{A}=\left[\begin{array}{ccccc}
0 & 1 & 0 & \cdots & 0 \\
0 & 0 & 1 & \cdots & 0 \\
\vdots & \vdots & \vdots & \ddots & \vdots \\
0 & 0 & 0 & \cdots & 1 \\
a_{1} & a_{2} & a_{3} & \cdots & \underline{a}_{n}
\end{array}\right] \text { is any Hurwitz matrix, } \\
& \boldsymbol{A} \in R^{n \times n} \\
& \text { iv) } \boldsymbol{w}=\left[\begin{array}{lllll}
0 & \cdots & 0 & 1
\end{array}\right]^{T} \in R^{n} . \\
& \text { v) } T^{\prime}\left(x, k, \widetilde{a}_{i}, \boldsymbol{b}_{i}, t\right) \text { is a } n^{\times n} \text { matrix which can be }
\end{aligned}
$$
computed as (8-1) and (8-2). 
International Journal of Fuzzy Logic and Intelligent Systems, vol. 6, no. 1, March 2006

$$
\begin{gathered}
\widehat{\Gamma}\left(x, k, \bar{a}_{i}, \delta_{i}, t\right)=\hat{\gamma} w^{T} \\
\hat{\gamma} \in R^{n} \\
\hat{\gamma}=\sum_{i=1}^{r} h_{i}(x)\left\{\frac{k x^{T}\left(a_{i}+R \Delta a-\underline{a}\right)}{k^{T} k}\right. \\
\left.+\left(b_{i}+\Delta b S\right) x\right\}+\frac{\operatorname{sgn}\left(x_{n}\right) d k}{k^{T} k}
\end{gathered}
$$

In (8-2), $\underline{a}$ is the transpose vector of the last row of A . That is,

$$
\underline{a}=\left[\begin{array}{lllll}
\underline{a_{1}} & \underline{a_{2}} & \underline{a_{3}} & \cdots & \underline{a_{n}}
\end{array}\right]^{T} \in R^{n} \text {. }
$$

Also, $R$ and $S$ are the diagonal matrixes defined as follows.

$R \equiv\left[\begin{array}{ccccc}\operatorname{sgn}\left(x_{1} x_{n}\right) & 0 & \cdots & 0 & 0 \\ 0 & \operatorname{sgn}\left(x_{2} x_{n}\right) & \cdots & 0 & 0 \\ \vdots & \vdots & \ddots & \vdots & \vdots \\ 0 & 0 & \cdots & \operatorname{sgn}\left(x_{n-1} x_{n}\right) & 0 \\ 0 & 0 & \cdots & 0 & \operatorname{sgn}\left(x_{n} x_{n}\right)\end{array}\right]$

$S \equiv\left[\begin{array}{ccccc}\operatorname{sgn}\left(k_{1} x_{1} x_{n}\right) & 0 & \cdots & 0 & 0 \\ 0 & \operatorname{sgn}\left(k_{2} x_{2} x_{n}\right) & \cdots & 0 & 0 \\ \vdots & \vdots & \ddots & \vdots & \vdots \\ 0 & 0 & \cdots & \operatorname{sgn}\left(k_{n-1} x_{n-1} x_{n}\right) & 0 \\ 0 & 0 & \cdots & 0 & \operatorname{sgn}\left(k_{n} x_{n} x_{n}\right)\end{array}\right]$

sgn in (9-1) and (9-2) is the sign function:

$$
\operatorname{sgn}(x)=+1 \quad \text { if } x>0
$$$$
\operatorname{sgn}(x)=-1 \quad \text { if } x<0
$$

Substituted in (6-1) - (6-3), the adaptive control laws (7-1) - (7-4) give the following closed loop dynamic equation.

$$
\begin{gathered}
\dot{x}=\underline{A} x+\boldsymbol{I}^{T}(x, k, t) k \\
k=-\Lambda \hat{T}\left(x, k, \widetilde{a}_{i}, \bar{b}_{i}, t\right) P \boldsymbol{P} \\
\tilde{a}_{i}^{T}=-\alpha_{1} h_{i}(x) x^{T} P w x^{T} \\
\tilde{\sigma}_{i}=-\alpha_{2} h_{i}(x) x^{T} P w k^{T} x
\end{gathered}
$$

where $\quad \tilde{a}_{i}=-\dot{a}_{i}, \tilde{b}_{i}=-b_{i}$.

$\Gamma(x, k, t)$ is a $n \times n$ matrix which can be computed as (11-1) and (11-2).

$$
\begin{gathered}
\Gamma(x, k, t)=\gamma w^{T} \gamma \in R^{n} \\
\gamma=\sum_{i=1}^{r} h_{i}(x)\left\{\frac{k x^{T}\left(a_{i}^{*}+\Delta a(t)-\underline{a}\right)}{k^{T} k}+\left(b_{i}^{*}+\Delta b(t)\right) x\right\}
\end{gathered}
$$

Theorem 1 : For the above closed loop dynamic equation $(10-1)-(10-4)$,

$$
\text { if } P w=p_{n n} w, p_{n n}>0
$$

is satisfied, then the following statements hold.

(a) the equilibrium point $\left(x, k, \widetilde{a}_{i}, \widetilde{b}_{i}\right)=0$ is uniformly stable.

(b) $x, k, \widetilde{a}_{i}, \widetilde{b}_{i}$ are uniformly bounded $\forall t \geq t_{0}$, $\forall \boldsymbol{x}\left(t_{0}\right) \Subset R^{n}, \forall \boldsymbol{k}\left(t_{0}\right) \in R^{p}, \forall \widetilde{\boldsymbol{a}}_{\boldsymbol{i}}\left(t_{0}\right) \in R^{n}$, $\forall b_{i}\left(t_{0}\right) \in R$.

(c) $\lim _{t \rightarrow \infty}\|x(t)\|=0, \quad \forall x\left(t_{0}\right) \in R^{n}, \quad \forall \boldsymbol{k}\left(t_{0}\right)$ $\in R^{p}, \forall \widetilde{a}_{i}\left(t_{0}\right) \in R^{n}, \forall \widetilde{b}_{i}\left(t_{0}\right) \in R$.

Proof : Let us choose a Lyapunov function

$$
V=x^{T} P x+k^{T} \Lambda^{-1} k+\frac{1}{\alpha_{1}} \sum_{i=1}^{r} \widetilde{a}_{i}{ }^{T} \widetilde{a}_{i}+
$$

$\frac{1}{\alpha_{2}} \sum_{i=1}^{r} \sigma_{i}^{2}$

The time derivative of $V$ is

$$
\begin{aligned}
V= & x^{T}\left(\boldsymbol{A}^{T} \boldsymbol{P}+\boldsymbol{P} \underline{\boldsymbol{A}}\right) \boldsymbol{x}+2 \boldsymbol{x}^{T} \boldsymbol{P} \boldsymbol{\Gamma}^{T} \boldsymbol{k}-2 \boldsymbol{k}^{T} \Lambda^{-1} \Lambda \boldsymbol{T P} \boldsymbol{x} \\
& +\frac{2}{\alpha_{1}} \sum_{i=1}^{r} \tilde{a}_{i}^{T} \widetilde{a}_{i}+\frac{2}{\alpha_{2}} \sum_{i=1}^{r} \boldsymbol{b}_{i} \boldsymbol{b}_{i}
\end{aligned}
$$

Now Since $-2 k^{T} \Lambda^{-1} \Lambda T P x$ is scalar

$-2 k^{T} \Lambda^{-1} \Lambda T P x=\left(-2 k^{T} \Lambda^{-1} \Lambda T P x\right)^{T}$

Therefore $V$ becomes

$$
\begin{aligned}
& V=-x^{T} \boldsymbol{Q} x+2 \boldsymbol{x}^{T} \boldsymbol{P}\left(\boldsymbol{\Gamma}^{T}-\boldsymbol{T}^{T}\right) k \\
& +\frac{2}{\alpha_{1}} \sum_{i=1}^{\gamma} \tilde{\boldsymbol{a}}_{i}^{T} \tilde{\boldsymbol{a}}_{i}+\frac{2}{\alpha_{2}} \sum_{i=1}^{r} \tilde{b}_{i} \boldsymbol{b}_{i} \\
& =-x^{T} Q x+2 x^{T} P w\left(\gamma^{T}-\hat{\gamma}^{T}\right) k \\
& +\frac{2}{\alpha_{1}} \sum_{i=1}^{r} \tilde{a}_{i}^{T} \tilde{\boldsymbol{a}}_{i}+\frac{2}{\alpha_{2}} \sum_{i=1}^{r} \tilde{b}_{i} \bar{b}_{i}
\end{aligned}
$$

From the adaptive laws in (10-3) and (10-4) and $\widehat{\gamma}$ and $\gamma$ in (8-2) and (11-2), we obtain

$$
\begin{aligned}
& V=-x^{T} Q x \\
& +2 x^{T} \boldsymbol{P} w \sum_{i=1}^{r} h_{i}(x)\left\{\frac{{\widetilde{\boldsymbol{a}_{i}}}_{i}^{T} x k^{T}}{k^{T} k}+\tau_{i} x^{T}\right\}_{k} \\
& +2 x^{T} P w \sum_{i=1}^{r} h_{i}(x)\left\{\frac{(\Delta a(t)-R \Delta a)^{T} x k^{T}}{k^{T} k}\right. \\
& +2 x^{T} P w \frac{\left\{d(t)-\operatorname{sgn}\left(x_{n}\right) d\right\} k^{T}}{k^{T} k} k \\
& -2 \sum_{i=1}^{r} h_{i}(x) x^{T} P w x^{T} \widetilde{a}_{i}-2 \sum_{i=1}^{r} h_{i}(x) x^{T} P w k^{T} x \\
& \text { After cancellation, } V \text { becomes } \\
& \begin{aligned}
V= & -x^{T} Q x \\
& +2 x^{T} P w \sum_{i=1}^{r} h_{i}(x)(\Delta a(t)-R \Delta a)^{T} x \\
+ & 2 x^{T} P w \sum_{i=1}^{r} h_{i}(x)(\Delta b(t)-\Delta b S) x^{T} k \\
+ & 2 x^{T} P w\left\{d(t)-\operatorname{sgn}\left(x_{n}\right) d\right\}
\end{aligned}
\end{aligned}
$$

From the condition of $P(12)$ and the definition of $R$ (9-1) and $S(9-2)$, the following inequalities hold. (refer to Appendix) 


$$
\begin{aligned}
& 2 x^{T} P w \sum_{i=1}^{r} h_{i}(x)(\Delta a(t)-R \Delta a)^{T} x \leq 0 \\
& 2 x^{T} P w \sum_{i=1}^{r} h_{i}(x)(\Delta b(t)-\Delta b S) x^{T} k \leq 0
\end{aligned}
$$

$2 x^{T} P w\left\{d(t)-\operatorname{sgn}\left(x_{n}\right) d\right\} \leq 0$

Thus, $\nabla$ in $(15)$ is bounded as follows.

$$
V \leq-x^{T} Q x
$$

According to the Lyapunov's Theorem, statement (a) is proved. Since $V$ is radially unbounded, statement (b) also is proved. (statement (b) implies $x \in L_{\infty}$.)

From (8-1) and (11-1), we can easily verify that $\widehat{T}\left(x, k, \widetilde{a}_{i}, \tilde{b}_{i}, t\right)$ and $\Gamma(x, k, t)$ are bounded for every $\left(x, k, \widetilde{a}_{i}, \widetilde{b}_{i}\right)$ bounded. From (10-1) - (10-4), since $x, k, \widetilde{a}_{i}, \bar{b}_{i}, \Gamma(x, k, t), \quad T\left(x, k, \widetilde{a}_{i}, \widetilde{b}_{i}, t\right)$ are bounded, it follows that $\dot{x}$ is bounded. ( $\bar{x} \in L_{\infty}$ )

Let $\lambda_{\min }(\boldsymbol{Q})$ denote the smallest eigenvalues of $\boldsymbol{Q}$. Then, since $Q$ is a positive definite matrix, the following property holds.

$\lambda_{\min }(Q)>0$ and $\lambda_{\min }(Q)|x|^{2} \leq x^{T} \boldsymbol{Q} x$

From this property, (17) becomes

$$
V \leq-x^{T} Q x \leq-\lambda_{\min }(Q)|x|^{2}
$$

Dividing both sides of (18) by $\lambda_{\min }(\boldsymbol{Q})$ and integrating them with respect to time, we obtain

$$
\begin{array}{r}
\int_{t_{0}}^{t}|x(\tau)|^{2} d \tau \leq-\frac{1}{\lambda_{\min }(\boldsymbol{Q})} \int_{t_{0}}^{t} V(\tau) d \tau \\
=\frac{1}{\lambda_{\min }(\boldsymbol{Q})}\left(V\left(t_{0}\right)-V(t)\right)
\end{array}
$$

which implies

$\lim _{t \rightarrow \infty} \int_{t_{0}}^{t}|x(\tau)|^{2} d \tau \leq \frac{1}{\lambda_{\min }(\boldsymbol{Q})}\left(V\left(t_{0}\right)-V(\infty)\right)<\infty$

Thus, we have $x \in L_{2}$.

Applying Barbalat's Lemma to $x(t)$ ( [16]: if $x \in L_{2} \cap L_{\infty} \quad$ and $\quad \bar{x} \in L_{\infty}$, then $\lim _{t \rightarrow \infty}\|x(t)\|$

$=0$ ), we conclude that $\lim _{t \rightarrow \infty}\|x(t)\|=0$, which proves statement (c).

From Theorem 1, we can conclude that the proposed adaptive fuzzy control algorithm can regulate the uncertain nonlinear system with the bounded control input and the parameter approximation error.

Remark : If we choose $\boldsymbol{P}=\boldsymbol{I}$, the Lyapunov equation becomes

$$
\underline{A}^{T}+\underline{A}=-Q
$$

If $A$ is a negative definite matrix, then the above Lyapunov equation (19) holds.

Thus, if $\underline{a} \in R^{n}$ is chosen to satisfy that

$$
\begin{aligned}
& \underline{A}=\left[\begin{array}{ccccc}
0 & 1 & 0 & \cdots & 0 \\
0 & 0 & 1 & \cdots & 0 \\
\vdots & \vdots & \vdots & \ddots & \vdots \\
0 & 0 & 0 & \cdots & 1 \\
a_{1} & \underline{a_{2}} & \underline{a_{3}} & \cdots & \underline{a_{n}}
\end{array}\right] \text { is a negative definite matrix , } \\
& \underline{\boldsymbol{A}} \in R^{n \times n}
\end{aligned}
$$

then the condition of $P(12)$ can be easily satisfied.

\section{Conclusion}

A new adaptive fuzzy control method is proposed to regulate the uncertain SISO nonlinear system. The proposed method has the dynamic state feedback structure with the adaptive fuzzy system identification.

Until now, most of the adaptive fuzzy control algorithms have been based on the feedback linearization method. Unlike the conventional feedback linearization based methods, the proposed algorithm does not require any assumption on the state variable. Thus, the proposed method can guarantee not only the local stability but also the global stability. Also, the proposed method can guarantee the boundedness of both the control input and the parameter approximation error directly without the additional projection algorithm. Simulation results have confirmed that the proposed algorithm could achieve the regulation problem of uncertain nonlinear systems.

\section{References}

[1] M. Sugeno, Industrial applications of fuzzy control, Elsevier Science, Amsterdam, 1985

[2] C. C. Lee, Fuzzy logic in control: Fuzzy logic controller-Part I and Part II, IEEE Trans. Syst., Man, Cybern., vol.20, no.2, pp.404-435, March, 1990

[3] B. Kosko, Neural networks and fuzzy systems, Prentice-Hall, Englewood Cliffs, 1992

[4] M. Jamshidi, N. Vadiee and T. J. Ress, Fuzzy logic and control, Prentice-Hall, Englewood Cliffs, 1993

[5] T. Takagi and M. Sugeno, Fuzzy identification of systems and its application to modeling and control, IEEE Trans. Syst., Man, Cybern., vol.15, no.1, pp.116-132, Jan. 1985

[6] S. G. Kong and B. Kosko, Adaptive fuzzy systems for backing up a truck and trailer, IEEE Trans. Neural Networks, vol.3, no.2, pp.211-223, March, 1992

[7] C. -Y. Su and Y. Stepanenko, Adaptive control of a class of nonlinear systems with fuzzy logic, IEEE Trans. Fuzzy Syst., vol.2, no.3, pp.285-294, Nov. 1994

[8] C. C. Wong, B. C. Huang and J. Y. Chen, Rule regulation of indirect adaptive fuzzy controller design, IEE Proc. Control Theory Appli., vol.145, no.6, pp.513-518, Nov. 1988 
[9] L. X. Wang, Stable adaptive fuzzy control of nonlinear systems, IEEE Trans. Fuzzy 'Syst., vol.1, no.2, pp.146-155, May 1993

[10] L. X. Wang, Adaptive fuzzy systems and control: Design and stability analysis, Englewood Cliffs, Prentice-Hall, 1993

[11] J. T. Spooner and K. M. Passino, Stable adaptive control using fuzzy systems and neural networks, IEEE Trans. Fuzzy Syst., vol.4, no.3, pp.339-359, Aug. 1996

[12] B. -S. Chen, C. -H. Lee and Y. -C. Chang, $H^{\infty}$ Tracking design of uncertain nonlinear SISO systems: Adaptive fuzzy approach, IEEE Trans. Fuzzy Syst., vol.4, no.1, pp.32-43, Feb. 1996

[13] K. Fischle and D. Schroder, An improved stable adaptive fuzzy control method, IEEE Tans. Fuzzy Syst., vol.7, no.1, pp.27-40, Feb. 1999

[14] D. L. Tsay, H. Y. Chung and C. J. Lee, The adaptive control of nonlinear systems using the Sugeno-type of fuzzy logic, IEEE Tans. Fuzzy Syst., vol.7, no.2, pp.225-229, Apr. 1999

[15] R. Ordonez and K. M. Passino, Stable multi-input multi-output adaptive fuzzy/neural control, IEEE Tans. Fuzzy Syst., vol.7, no.3, pp.345-353, June 1999

[16] S. S. Sastry and A. Isidori, Adaptive control of linearizable systems, IEEE Trans. Automat. Contr., vol.34, no.11, pp.1123-1131, Nov. 1989

[17] R. H. Cannon, Dynamics of physical systems, McGrawHill, New York, 1967

[18] F. C. Sun, Z. Q. Sun and G. Feng, An adaptive fuzzy controller based on sliding mode for robot manipulators, IEEE Trans. Syst., Man, Cybern., vol.29, no.5, pp.661-667, Oct. 1999

[19] Y. G. Leu, T. T. Lee and W. Y. Wang, Observer based adaptive fuzzy-neural control for unknown nonlinear dynamical systems, IEEE Trans. Syst., Man, Cybern., vol.29, no.5, pp.583-591, Oct. 1999

[20] R. M. Sanner and J. -J. E. Slotine, Gaussian networks for direct adaptive control, IEEE Trans. Neural Networks, vol.3, no.6, pp.837-863, Nov. 1992

[21] K. S. Narendra and A. M. Annaswamy, Stable adaptive systems, Prentice-Hall, Englewood Cliffs, 1989

[22] G. A. Rovithakis and M. A. Christodoulou, Adaptive control of unknown plants using dynamic neural networks, IEEE Trans. Syst., Man, Cybern., vol. 24, no.3, pp. 400-412, March 1994

[23] P. A. Ioannou and J. Sun, Robust adaptive control, Prentice Hall, New Jersey, 1996

\section{Appendix}

\section{Proof of Inequality (16-1) :}

Using $\sum_{i=1}^{r} h_{i}(x)=1$, the condition of $P(12)$ and the definition of $\boldsymbol{R}(9-1)$,

$$
2 \boldsymbol{x}^{T} \boldsymbol{P} \boldsymbol{w} \sum_{i=1}^{r} h_{i}(x)(\Delta a(t)-\boldsymbol{R} \Delta a)^{T} x
$$

$$
\begin{aligned}
= & 2 \boldsymbol{x}^{T} p_{n n} \boldsymbol{w}(\Delta a(t)-\boldsymbol{R} \Delta \boldsymbol{a})^{T} \boldsymbol{x} \\
& =2 p_{n n}\left(\Delta a_{1}(t)-\operatorname{sgn}\left(x_{1} x_{n}\right) \Delta a_{1}\right) x_{1} x_{n} \\
& +2 p_{n n}\left(\Delta a_{2}(t)-\operatorname{sgn}\left(x_{2} x_{n}\right) \Delta a_{2}\right) x_{2} x_{n} \\
+ & \cdots+2 p_{n n}\left(\Delta a_{n}(t)-\operatorname{sgn}\left(x_{n} x_{n}\right) \Delta a_{n}\right) x_{n} x_{n} \\
& =2 p_{n n}\left(x_{1} x_{n} \Delta a_{1}(t)-\left|x_{1} x_{n}\right| \Delta a_{1}\right) \\
& +2 p_{n n}\left(x_{2} x_{n} \Delta a_{2}(t)-\left|x_{2} x_{n}\right| \Delta a_{2}\right) \\
+ & \cdots+2 p_{n n}\left(x_{n} x_{n} \Delta a_{n}(t)-\left|x_{n} x_{n}\right| \Delta a_{n}\right) \leq 0
\end{aligned}
$$

\section{Proof of Inequality (16-2) :}

Using $\sum_{i=1}^{r} h_{i}(x)=1$, the condition of $P(12)$ and the definition of $S(9-2)$,

$$
\begin{aligned}
& 2 x^{T} P w \sum_{i=1}^{r} h_{i}(x)(\Delta b(t)-\Delta b S) x^{T} k \\
= & 2 x^{T} p_{n n} w(\Delta b(t)-\Delta b S) x^{T} k \\
= & 2 p_{n n}\left(\Delta b(t)-\operatorname{sgn}\left(k_{1} x_{1} x_{n}\right) \Delta b\right) k_{1} x_{1} x_{n} \\
& +2 p_{n n}\left(\Delta b(t)-\operatorname{sgn}\left(k_{2} x_{2} x_{n}\right) \Delta b\right) k_{2} x_{2} x_{n} \\
& +\cdots+2 p_{n n}\left(\Delta b(t)-\operatorname{sgn}\left(k_{n} x_{n} x_{n}\right) \Delta b\right) k_{n} x_{n} x_{n} \\
= & 2 p_{n n}\left(k_{1} x_{1} x_{n} \Delta b(t)-\left|k_{1} x_{1} x_{n}\right| \Delta b\right) \\
& +2 p_{n n}\left(k_{2} x_{2} x_{n} \Delta b(t)-\left|k_{2} x_{2} x_{n}\right| \Delta b\right) \\
+ & \cdots+2 p_{n n}\left(k_{n} x_{n} x_{n} \Delta b(t)-\left|k_{n} x_{n} x_{n}\right| \Delta b\right) \leq 0
\end{aligned}
$$

Proof of Inequality (16-3) :

$$
\begin{aligned}
2 & x^{T} P w\left\{d(t)-\operatorname{sgn}\left(x_{n}\right) d\right\} \\
& =2 p_{n n}\left(d(t)-\operatorname{sgn}\left(x_{n}\right) d\right) x_{n} \\
& =2 p_{n n}\left(x_{n} d(t)-\left|x_{n}\right| d\right) \leq 0
\end{aligned}
$$

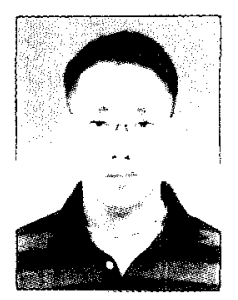

\section{Chang-Woo Park}

received the B.S. degree in electronics from Korea University in 1997 and M.S. and $\mathrm{Ph} . \mathrm{D}$. degrees in electronics from Yonsei University, Seoul, Korea, in 1999 and 2003, respectively. $\mathrm{He}$ has been a senior researcher in Korea Electronics Technology Institute under the Ministry of Commerce, Industry and Energy. His current research interests include intelligent system, fuzzy system and robotics.

$\begin{array}{ll}\text { Phone } & :+82-32-621-2854 \\ \text { Fax } & :+82-32-621-2866 \\ \text { E-mail } & : \text { drcwpark@keti.re.kr }\end{array}$




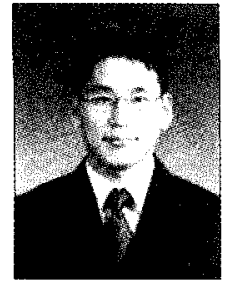

Jun-Hyuk Choi

received the MS. degree in Power electronics from Sungkyunkwan University in 2003. He has been a researcher in Korea Electronics Technology Institute under the Ministry of Commerce, Industry and Energy. His current research interests are in the fields of power electronics, electrical

drives.

$\begin{array}{ll}\text { Phone } & :+82-32-621-2859 \\ \text { Fax } & :+82-32-621-2855 \\ \text { E-mail } & : \text { cjh@keti.re.kr }\end{array}$

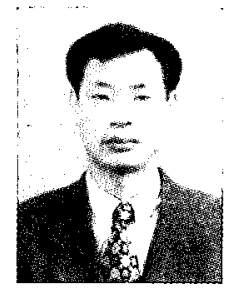

Ha-Gyeong Sung

Sung received the B.S. and M.S. degrees in mechanical engineering from Hanyang University in 1986 and 1995 respectively and received Ph.D. degree in mechanical engineering from Ajou University, Korea, in 2003. He has been a chair of center in Korea Electronics Technology Institute under the Ministry of Commerce, Industry and Energy. His current research interests include human computer interface, motor design and tele-operating system.

$\begin{array}{ll}\text { Phone } & :+82-32-621-2841 \\ \text { Fax } & :+82-32-621-2855 \\ \text { E-mail } & : \text { sunghg@keti.re.kr }\end{array}$

